\title{
Pairing Calculation on Supersingular Genus 2 Curves
}

\author{
Colm Ó hÉigeartaigh and Michael Scott \\ School of Computing, Dublin City University. \\ Ballymun, Dublin 9, Ireland \\ \{coheigeartaigh, mike\}@computing.dcu.ie
}

\begin{abstract}
In this paper we describe how to efficiently implement pairing calculation on supersingular genus 2 curves over prime fields. We find that, contrary to the results reported in [8], pairing calculation on supersingular genus 2 curves over prime fields is efficient and a viable candidate for the practical implementation of pairing-based cryptosystems. We also show how to eliminate divisions in an efficient manner when computing the Tate pairing, assuming an even embedding degree, and how this algorithm is useful for curves of genus greater than one.
\end{abstract}

Keywords: Tate pairing, hyperelliptic curves, pairing computation.

\section{Introduction}

Following a seminal paper by Boneh and Franklin [5] in 2001, there has been an explosion of interest in the exploitation of the properties of bilinear pairings on elliptic curves for cryptographic protocols. Naturally, there has also been much focus on the efficient implementation of pairings. Victor Miller gave the first algorithm 20121] for computing a bilinear pairing, specifically the Weil pairing. However in practice the Tate pairing is used, as it is computationally less expensive.

In an important paper, Barreto et al. 2] gave criteria under which divisions in Miller's algorithm can be eliminated entirely. According to 25, this reduces the calculation time by almost $50 \%$. Other papers which describe important improvements to computing the Tate pairing on elliptic curves are [12] and [3]. Numerous papers describe the actual implementational details, for example see [26].

Although the vast majority of work has been done using elliptic curves, an increasing amount of attention is being focused on computing pairings using hyperelliptic curves of genus 2. Choie and Lee [8] investigate the implementation of the Tate pairing on supersingular genus 2 curves of embedding degree 4, over large prime fields. Barreto et. al. [1] describe an efficient implementation of the Tate pairing using the eta pairing construct on supersingular genus 2 curves, over fields of characteristic 2. The significance of this paper is that it not only shows that pairing computation is comparable on genus 2 curves to elliptic curves, but that it can in fact be even faster. 
In this paper, we first of all give an improvement to Miller's algorithm for calculating the Tate pairing for arbitrary curves of even embedding degree. This algorithm is more efficient than the GHS algorithm [12, but not as efficient as the denominator elimination technique of Barreto et. al 2 in the general case. However, we show that the new algorithm is more efficient than the denominator elimination algorithm for special cases using curves of genus greater than one.

We then report an efficient implementation of the Tate pairing on genus 2 curves over prime fields. We detail various enhancements to Miller's algorithm that are in the literature, explaining how to apply them to the genus 2 case using prime fields for the first time. We give more efficient formulae for computing the functions required in Miller's algorithm for the genus 2 case than that reported in 8 , the saving being a squaring and a multiplication in each iteration. Finally, we report timings for computing the Tate pairing, that establishes new benchmarks for pairing implemention on genus 2 curves over large prime fields.

This paper is organised as follows. Section 2 gives an overview of the Tate pairing and Miller's Algorithm. Section 3 details an algorithm for computing the Tate pairing, assuming an even embedding degree, without using the two-variable approach of 12. Section 4 shows how to apply various techniques from the literature for speeding up pairing computation to the specific genus 2 case over prime fields. Section 5 gives experimental results, and we draw our conclusions in section 6 .

Section 3 was partly presented in a short paper on the ePrint archive (see [15), and was presented in the rump session at the ECC 2005 conference. We note that some of the ideas in this section were derived independently in Kobayashi et al. [17.

\section{The Tate Pairing}

We say that a subgroup of the degree zero divisor class group of a hyperelliptic curve $C$ defined over a finite field $\mathbb{F}_{p}$ has embedding degree $k$, if the order $r$ of the subgroup divides $p^{k}-1$, but does not divide $p^{i}-1$ for any $0<i<k$. The Tate pairing maps the discrete logarithm in the subgroup to the discrete logarithm in the finite field $\mathbb{F}_{p^{k}}$, which is the basis of the Frey-Rück attack [1].

Using the notation above, let $r$ be a prime number which is coprime to $p$. Let $G=J_{C}\left(\mathbb{F}_{p^{k}}\right)$ be the Jacobian Variety of $C$ over $\mathbb{F}_{p^{k}}$, which is isomorphic to the degree zero divisor class group, and let $G[r]$ be the $r$-torsion group and $G / r G$ the quotient group. Then the Tate pairing is defined as;

$$
\langle\cdot, \cdot\rangle_{r}: G[r] \times G / r G \rightarrow \mathbb{F}_{p^{k}}^{*} /\left(\mathbb{F}_{p^{k}}^{*}\right)^{r}
$$

We follow Galbraith et al. 12 in defining the first argument over the smaller field $\mathbb{F}_{p}$ instead of $\mathbb{F}_{p^{k}}$. This greatly improves computational efficiency, as all the coefficients of the functions in Miller's Algorithm will then also be defined over the field $\mathbb{F}_{p}$. To allow for an efficient implementation of the extension field arithmetic, we also assume that the embedding degree $k$ is even. The Tate pairing 
as detailed above is only defined up to $r$-th powers. As a unique value is required for cryptographic purposes, we define the reduced pairing;

$$
\left\langle D_{1}, D_{2}\right\rangle_{r}^{\left(p^{k}-1\right) / r}
$$

The Tate pairing is both well-defined and non-degenerate. However its most important property is bilinearity. We define bilinearity for any integer $n$ as, $\langle[n] P, Q\rangle \equiv\langle P,[n] Q\rangle \equiv\langle P, Q\rangle^{n}$ (modulo $r^{t h}$ powers). The Tate pairing can be computed using an algorithm due to Miller [20]21, as described in Algorithm 1 for an arbitrary hyperelliptic curve. This algorithm is basically the usual "double and add" algorithm combined with an evaluation of certain intermediate functions (see chapter 9 of 4 . for more details).

In Algorithm 1 the divisions are postponed until the end of the loop, to avoid performing a division each loop iteration. To do this we use two variables in the loop, to effectively replace a division with a squaring each loop iteration, which is considerably less expensive to compute. This is an idea given by Galbraith et. al. [12]. However as we shall see in the next section, when the embedding degree is even, this optimisation is unnecessary.

After the main loop, the final exponentiation of $\left(p^{k}-1\right) / r$ is performed to obtain a unique value over $\mathbb{F}_{p^{k}}$. It is this unique value which can then be used for cryptographic purposes. It is well known that if arithmetic in $\mathbb{F}_{p^{k}}$ is implemented using quadratic extensions, an element in this field can be exponentiated to the power of $p^{k / 2}$ using a simple conjugation in $\mathbb{F}_{p^{k}}$ over $\mathbb{F}_{p^{k / 2}}$. Conjugation in the quadratic extension is denoted by $\bar{x}=(a-b i)$ for an element $x=(a+b i) \in \mathbb{F}_{p^{k}}$.

Taking advantage of this, it is standard to efficiently compute the final exponentiation as $f=\bar{f} / f$ followed by $f=f^{\left(p^{k / 2}+1\right) / \phi_{k}(p)}$ and $f=f^{\left(\phi_{k}(p) / r\right)}$, where $\phi_{d}(x)$ is the $d^{t h}$ cyclotomic polynomial. The final $f=f^{\left(\phi_{k}(p) / r\right)}$ exponentiation can be computed efficiently using either Lucas Sequences 2829] or the multi-exponentiation approach [1614.

An important improvement on Miller's Algorithm as detailed above was given in 2 for elliptic curves. If the $x$-coordinate of the image point $Q$ is defined over a subfield of $\mathbb{F}_{p^{k}}$, then the denominator, or the $f_{d}$ variable in algorithm 1 , will also be defined over a subfield of $\mathbb{F}_{p^{k}}$. This is because the denominator function relies solely on the evaluation at the $x$-coordinate of $Q$ each iteration. As any value defined over a subfield of $\mathbb{F}_{p^{k}}$ will be annihilated by the final exponentiation of $\left(p^{k / 2}-1\right)$, the $f_{d}$ variable can be removed from algorithm 2 completely.

Some distortion maps naturally map the $x$-coordinate of a point to a subfield of $\mathbb{F}_{p^{k}}$. When this is not the case, a simple transformation of the point as detailed in the next section will have the desired effect. However, as will be seen, this approach is problematic for curves of genus greater than one.

\section{Eliminating Divisions in Miller's Algorithm}

In this section, we show how the denominator elimination technique is problematic with curves of genus greater than one, assuming that a suitable distortion 


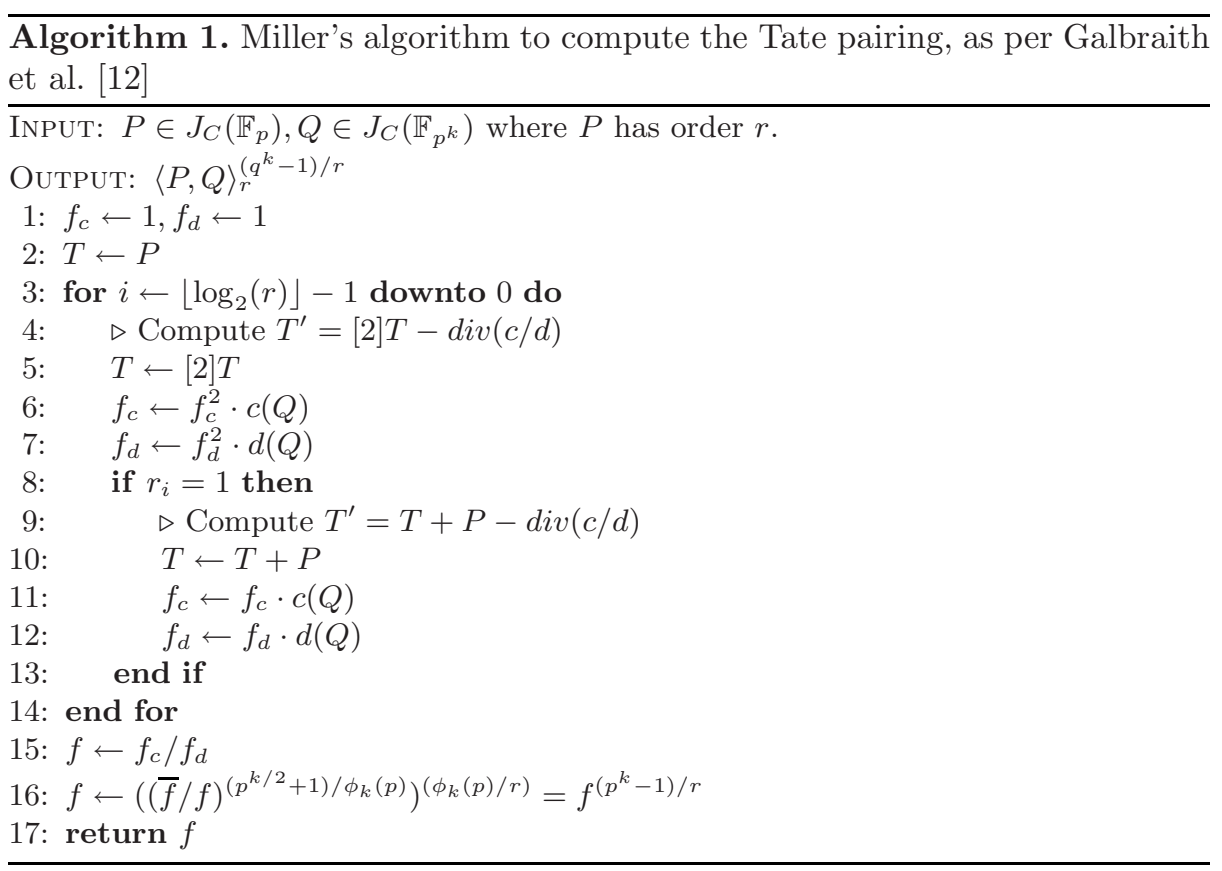

map does not exist. We then present a more efficient algorithm for computing the Tate pairing over quadratic extension fields than is given in algorithm 1 . which overcomes the problems associated with denominator elimination in certain contexts.

As seen in the previous section, another approach is required to get the denominator elimination technique to work, when no distortion map exists that maps the $x$-coordinate of a point from the ground field to a subfield of the field $\mathbb{F}_{p^{k}}$. Instead we must use an idea given in a paper by Barreto et. al. 3. To apply denominator elimination in this case, generate a "distorted point" $Q$ over $\mathbb{F}_{p^{k}}$ and get a tracezero point with: $R=Q-Q^{p^{k / 2}}$. The point $R$ will have an $x$-coordinate defined over a subfield, and so the denominator elimination technique can be used.

However, this technique is problematic when the genus is greater than one, as it increases the weight of the image divisor. For example, if we are evaluating at a degenerate divisor in the genus 2 setting, which consists of a single point on the support, then the above mapping will result in a more general divisor with two points. This will not happen using elliptic curves, as each class in the divisor class group can be represented by a divisor with a single point in the support. So in the genus 2 setting, instead of evaluating the functions in Miller's algorithm at a single point, they must be evaluated at two points to use the denominator elimination technique. This drastically reduces the efficiency of denominator elimination.

We now present an alternative way to proceed, by introducing a new variant of Miller's algorithm, assuming that the embedding degree of the curve is even, 
Table 1. Complexity of function calculation per iteration in Miller's Algorithm

\begin{tabular}{|l|l|l|}
\hline case & description & complexity \\
\hline 1 & Original Approach & $1 \mathrm{I}, 2 \mathrm{M}, 1 \mathrm{~S}$ \\
2 & Two-variable Approach & $2 \mathrm{M}, 2 \mathrm{~S}$ \\
3 & Algorithm 2 & $2 \mathrm{M}, 1 \mathrm{~S}$ \\
4 & Denominator Elimination & $1 \mathrm{M}, 1 \mathrm{~S}$ \\
\hline
\end{tabular}

as is almost always the case for practical implementations. The finite field $\mathbb{F}_{p^{k}}$ is then typically represented as a quadratic extension of $\mathbb{F}_{p^{k / 2}}$. It is well known that for an element $x=(a+b i) \in \mathbb{F}_{p^{k}}$, then $\left(\frac{1}{x}\right)^{p^{k / 2}-1}=(\bar{x})^{p^{k / 2}-1}$. This effectively replaces an expensive operation with one that is free to compute.

This technique is exploited by Scott [27] when computing the Weil pairing. Scott proposes exponentiating the pairing value to the power of $p^{k / 2}-1$, which means that the inversion in the Miller loop can be replaced with a conjugation. However, no one has observed that it is possible to use this idea when computing the Tate pairing, without any extra computation being involved. When computing the Tate pairing, the final exponentiation to obtain a unique $r^{\text {th }}$ root of unity includes the factor $\left(p^{k / 2}-1\right)$, as $\left(p^{k}-1\right) / r=\left(p^{k / 2}-1\right)\left(p^{k / 2}+1\right) / r$. So as the output of the loop is implicitly raised to the power of $\left(p^{k / 2}-1\right)$, there is no need of the strategy of using two variables to eliminate divisions, as a division in the main loop can be replaced by a multiplication and a conjugation. The new algorithm is described in Algorithm 2 .

As the variable $f_{d}$ is eliminated from the pairing calculation, the saving is a squaring over $\mathbb{F}_{p^{k}}$ each iteration of the loop compared to the GHS approach. This is still not as efficient as performing denominator elimination, which would save a multiplication over this again each iteration. However, when computing the Tate pairing with curves of genus greater than one, and using a distortion map that does not allow denominator elimination directly, algorithm 2 is a slightly more efficient algorithm.

The reason for this is that the denominator elimination algorithm consists of two evaluations at the line function each iteration (or one evaluation of a more complicated form if Mumford representation (see Cantor [6]) is used). Algorithm 2 consists of one evaluation at the line function, and one evaluation at the vertical line function, which requires less computation to evaluate than the line function. Algorithm 2 is also less restrictive than using denominator elimination, as it places no conditions on the form of the image divisor. Table 1 illustrates the complexity of the different algorithms in more detail.

\section{Implementing the Pairing}

In this section, various techniques that allow for an efficient implementation of the Tate pairing using supersingular genus 2 curves over prime fields are described. Timings are given in section 5 . 


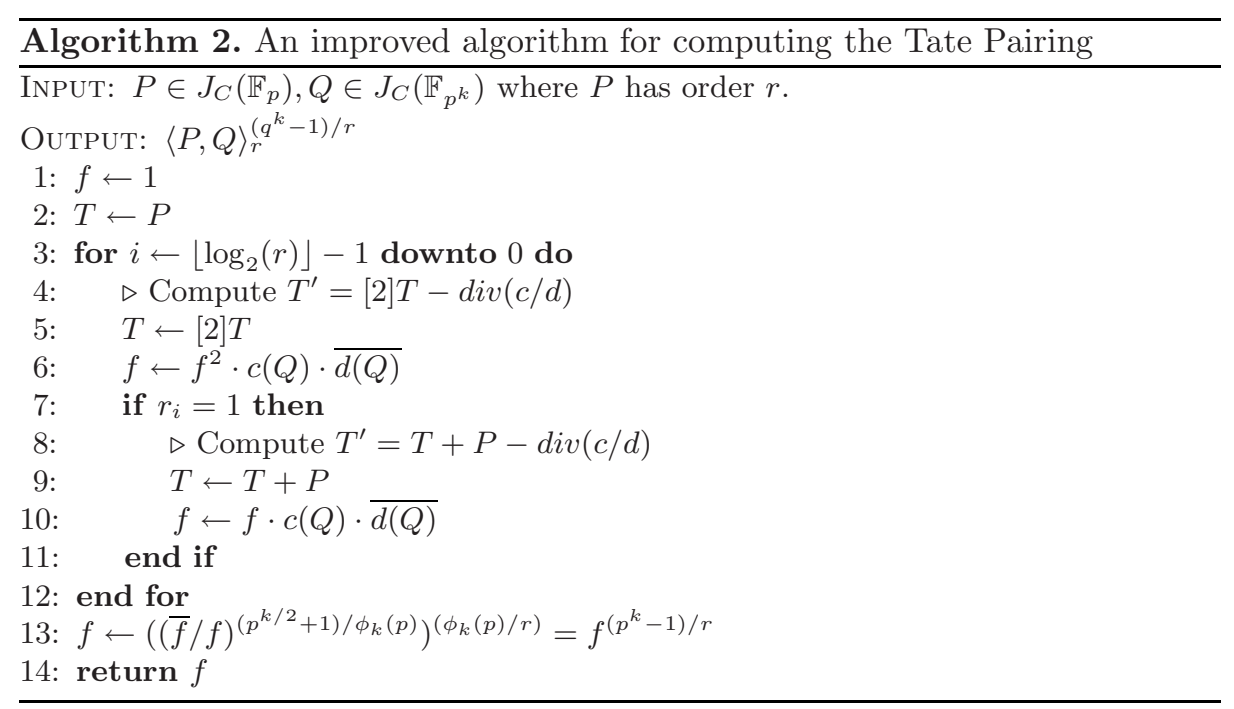

\subsection{The Curve}

Following [7] and [8], we implement the Tate pairing on the supersingular genus 2 curve;

$$
H: y^{2}=x^{5}+a, a \in \mathbb{F}_{p}^{*}, p \equiv 2,3 \bmod 5
$$

In practice, we take $a=1$ for convenience. The other supersingular genus 2 curve defined over a prime field with a low embedding degree that was given in [7], is unsuitable for cryptography as it is isogenous to a product of elliptic curves.

The order of the Jacobian of this curve is $\# J_{C}\left(\mathbb{F}_{p}\right)=p^{2}+1$, and hence the embedding degree of the curve is 4 . The distortion map that maps points on the curve defined over the field $\mathbb{F}_{p}$ to the larger field $\mathbb{F}_{p^{4}}$ is given as;

$$
\phi(x, y)=\left(\zeta_{5} x, y\right)
$$

where $\zeta_{5}$ is a primitive $5^{\text {th }}$ root of unity defined over $\mathbb{F}_{p^{4}}$. Note that $\zeta_{5}$ maps the $x$-coordinate to $\mathbb{F}_{p^{4}}$, and hence does not give denominator elimination directly. Choie and Lee give explicit formulae [8] for calculating the functions required for the genus 2 Tate pairing, which are derived from Lange's explicit genus 2 formulae [18] and Miyamoto et al.'s formulae [22].

Doubling a divisor is by far the most important part of the group arithmetic for pairings, under the assumption that we are using a prime-order subgroup which has an order of low Hamming Weight. In [8] the given cost of doubling a general divisor (in the overwhelmingly common case) and extracting the functions required for Miller's algorithm is 1 inversion, 23 multiplications and 5 squarings over $\mathbb{F}_{p}$. However, we save a squaring and a multiplication over this. In table 6 of the appendix, we give the formulae for doubling a general divisor as per [8], with these 
Table 2. Comparison of the cost of doubling in $J_{H}$

\begin{tabular}{|l|l|l|}
\hline & doubling & $\mathrm{l}(\mathrm{x})$ \\
\hline 22 & $1 \mathrm{I}, 23 \mathrm{M}, 4 \mathrm{~S}$ & $3 \mathrm{M}$ \\
\hline 18 & $1 \mathrm{I}, 22 \mathrm{M}, 5 \mathrm{~S}$ & $3 \mathrm{M}$ \\
\hline $\mathbf{8}$ & $1 \mathrm{I}, 23 \mathrm{M}, 5 \mathrm{~S}$ & no cost \\
our work & $1 \mathrm{I}, 22 \mathrm{M}, 4 \mathrm{~S}$ & no cost \\
\hline
\end{tabular}

optimisations built in (the multiplication is saved in step 8). Note our assumption that, as the characteristic of the field is odd, the $h$ polynomial is zero, where the $h$ polynomial comes from the definition of the curve as $y^{2}+h(x) y=f(x)$. Table 2 summarises the computational cost of doubling a general divisor.

We believe that the formulae in table 6 are optimal, as they have the same computational cost as simply doubling a divisor as given in [18, ie. calculating the functions required for Miller's algorithm is for free.

\subsection{Prime-Order Subgroup}

We use the conventions suggested by Lenstra and Verheul [19] and used by Scott [27, to define the levels of security required. For a more thorough comparison of security levels we refer the reader to Galbraith et al. 13]. Here the security levels are defined as $(160 / 1024),(192 / 2048)$ and $(224 / 4096)$, where the first number in each term is the group size, and the second number is the size of the field $\mathbb{F}_{p^{k}}$. As our embedding degree is $k=4$, we are required to work with finite fields $\mathbb{F}_{p}$, where $p \sim 256,512$ and 1024-bits.

When considering what group size to use there are two options, either to use a prime-order subgroup or the full order of the Jacobian. The latter has the advantage that the order of the Jacobian often has a small Hamming weight, and the final exponentiation can be far less expensive. However, if we choose the order of the prime-order subgroup such that it has a low Hamming weight, and if it is far smaller than the order of the Jacobian, then the former method is better.

Rather than using a random prime-order subgroup, we choose a special prime of low Hamming Weight known as a Solinas prime [30]. These primes require only two additions in Miller's algorithm. As Duursma and Lee noted 9], the final addition can be skipped assuming that denominator elimination is applied. See section 5 for further details on the parameters used.

\subsection{Finite Field Representation}

The best way to represent elements of the field $\mathbb{F}_{p^{4}}$ is to represent them as a quadratic extension of $\mathbb{F}_{p^{2}}$, which is in turn a quadratic extension of $\mathbb{F}_{p}$, as opposed to representing $\mathbb{F}_{p^{4}}$ as a quartic extension of $\mathbb{F}_{p}$. If the prime $p$ is congruent to $5 \bmod 8$, then the irreducible polynomial $x^{2}+2$ can be used to represent the quadratic extension field $\mathbb{F}_{p^{2}}$. So, assuming that $\beta=-2$ is a quadratic nonresidue, we represent elements of the field $\mathbb{F}_{p^{2}}$ as $(a+b \sqrt{\beta})$, where $a, b \in \mathbb{F}_{p}$, 
and we represent elements of the field $\mathbb{F}_{p^{4}}$ as $(c+d \sqrt[4]{\beta})$, where $c, d \in \mathbb{F}_{p^{2}}$. An advantage of using a prime $p \equiv 5 \bmod 8$ is the resulting simple formula for modular square roots, as required for generating points on the curve.

Using our representation, a multiplication of two elements in $\mathbb{F}_{p^{2}}$ is computed using the Karatsuba method as

$$
\begin{aligned}
(a+b \sqrt{\beta})(x+y \sqrt{\beta}) & =(a x-2 b y+(a y+b x) \sqrt{\beta}) \\
& =(a x-2 b y+((a+b)(x+y)-a x-b y) \sqrt{\beta})
\end{aligned}
$$

which takes $3 M$, where $M$ is a multiplication over $\mathbb{F}_{p}$. Similarly, a multiplication of two elements in $\mathbb{F}_{p^{4}}$ takes $9 M$. Squaring an element in $\mathbb{F}_{p^{2}}$ is computed as

$$
\begin{aligned}
(a+b \sqrt{\beta})^{2} & =\left(a^{2}-2 b^{2}+2 a b \sqrt{\beta}\right) \\
& =((a+b)(a-2 b)+a b+2 a b \sqrt{\beta})
\end{aligned}
$$

which takes $2 M$ - slightly cheaper than a general multiplication in $\mathbb{F}_{p^{2}}$. Similarly, squaring an element in $\mathbb{F}_{p^{4}}$ takes $6 M$, which is considerably cheaper than the $9 M$ required for a general multiplication in $\mathbb{F}_{p^{4}}$. We also note that our method for squaring an element in $\mathbb{F}_{p^{4}}$ is cheaper than the $8 M$ given by Choie and Lee [8].

\subsection{Using Degenerate Divisors}

Duursma and Lee 9] introduced the notion of working with a degenerate divisor for pairing applications with curves of genus greater than 1 . In the genus 2 context, we will define a degenerate divisor as a divisor with only one point in its support, rather than the more general two. There is no advantage to be gained in using a degenerate divisor as the first argument to Miller's algorithm, as with the first doubling the divisor will turn into a more general divisor with two points on the support, unless one takes advantage of an automorphism that keeps the divisor in its special shape (eg. see [1]).

However, as we are evaluating the second divisor at a function, we achieve a speedup by evaluating at a degenerate divisor (ie. a single point). Evaluating at a more general divisor requires evaluation at two points, or else using the divisor's Mumford representation. Pairing-based cryptosystems, such as the Identity-Based Encryption scheme of Boneh and Franklin [5], can be easily modified to take advantage of the form of degenerate divisors to speed up the encryption process. However, in this case the decryption process involves pairing two general divisors, so it is important to give timings for both cases. Frey and Lange [10] discuss in more detail in which cases the second argument to Miller's algorithm can be chosen to be a degenerate divisor.

\subsection{Evaluating Functions}

Each iteration of the loop requires the evaluation of the function $f_{c}=y_{1}-$ $\left(\left(x_{1} \zeta_{5}\right)^{3} s_{1}+\left(x_{1} \zeta_{5}\right)^{2} l_{2}+\left(x_{1} \zeta_{5}\right) l_{1}+l_{0}\right)$, where $s_{1}, l_{0}, l_{1}, l_{2}$ are from Cantor's algorithm, $\zeta_{5}$ is a primitive $5^{\text {th }}$ root of unity defined over $\mathbb{F}_{p^{4}}$ and $x_{1}$ is the $x$ coordinate of the point at which we are evaluating. As $\left(x_{1} \zeta_{5}\right)^{3},\left(x_{1} \zeta_{5}\right)^{2}$ and 
$\left(x_{1} \zeta_{5}\right)$ can be precomputed, this leaves 12 multiplications over $\mathbb{F}_{p}$ to be computed each time the function is evaluated. However, a multiplication may be saved by examining relations between various powerings of the $5^{\text {th }}$ root of unity.

If $\zeta_{n}$ is a primitive $n^{\text {th }}$ root of unity in a field $K$, then its conjugates over the prime subfield $K_{0}$ of $K$ are also primitive $n^{t h}$ roots of unity 23 . Also, $\zeta_{n}^{a}$ is a primitive $n^{t h}$ root of unity if and only if $a$ and $n$ are coprime. In our case, the third power of a $5^{\text {th }}$ primitive root of unity over $\mathbb{F}_{p^{4}}$ is related to the second power by conjugation: $\zeta_{5}^{3}=\overline{\zeta_{5}^{2}}$

So instead of evaluating an equation of the form $a+b \zeta_{5}+c \zeta_{5}^{2}+d \zeta_{5}^{3}$, where $b=$ $-x_{1} l_{1}, c=-x_{1}^{2} l_{2}$ and $d=-x_{1}^{3} s_{1}$, let $\zeta_{5}^{2}=(m+n \sqrt[4]{\beta})$ where $m, n \in \mathbb{F}_{p^{2}}$. Then we can compute $a+b \zeta_{5}+c \zeta_{5}^{2}+d \zeta_{5}^{3}$ as $a+b \zeta_{5}+((c+d) m,(c-d) n)$. Computing $c$ and $d$ takes only two multiplications over $\mathbb{F}_{p}$ (with a precomputation of 1 squaring and 1 multiplication). Computing $(c+d) m$ and $(c-d) n$ takes 4 multiplications, with a precomputation of 6 multiplications. Computing $b \zeta^{5}$ takes 4 multiplications, with a precomputation of 4 multiplications. Thus the total multiplication count in evaluating the function is 10 multiplications, a saving of two multiplications, with a precomputation of 11 multiplications and 1 squaring.

Evaluating the image point at the vertical line functions takes 8 multiplications over $\mathbb{F}_{p}$, assuming a precomputation of 6 multiplications. So the total cost of evaluating a point at the functions is 18 multiplications over $\mathbb{F}_{p}$ per iteration, with a precomputation of 1 squaring and 17 multiplications.

\subsection{Using Denominator Elimination}

As detailed in section 3 it is more efficient to use Algorithm 2 than denominator elimination, assuming a distortion map that does not give denominator elimination directly, and that the image divisor is a degenerate divisor. However, it is possible to reduce the performance gap by using customized multiplication routines, as detailed in this section.

Given a point $Q=(x, y) \in \mathbb{F}_{p^{4}}$, the transformation $R=Q-Q^{p^{2}}$ gives a divisor $R$ suitable for use with denominator elimination. Writing this as $R=(x, y)+$ $(\bar{x},-y)$ avoids using Cantor's algorithm over $\mathbb{F}_{p^{4}}$ and keeps the two points on the support of the divisor separate. A benefit of this approach is that the function calculated in the main doubling loop, $f_{c}=y_{1}-\left(\left(x_{1} \zeta_{5}\right)^{3} s_{1}+\left(x_{1} \zeta_{5}\right)^{2} l_{2}+\left(x_{1} \zeta_{5}\right) l_{1}-\right.$ $\left.l_{0}\right)$, can be reused for the calculation of the required function for the second point.

Let the function $f_{c}=((a+b \sqrt{\beta})+(c+d \sqrt{\beta}) \sqrt[4]{\beta})$ for the first point $(x, y)$. Then, for the second point $(\bar{x},-y)$, the function $f_{c}=((a-2 y+b \sqrt{\beta})-(c+d \sqrt{\beta}) \sqrt[4]{\beta})$. So the calculation of the second function is effectively for free, as it simply involves two subtractions and a conjugation using the function generated by the first point. However, we still have to multiply the two functions together.

In each iteration of the loop, the function $f_{c}$ is evaluated twice, ie. at the two points. As seen above, the two functions are closely related. It is possible to exploit these relations to speed-up the calculation. So instead of calculating each function separately and multiplying them separately by the overall accumulating variable, we multiply the functions $f_{c_{1}}$ and $f_{c_{2}}$ together first, before multiplying the result with the accumulating function. 
Normally, multiplying two general elements in $\mathbb{F}_{p^{4}}$ can be done with only 9 multiplications over $\mathbb{F}_{p}$, using the Karatsuba technique. For the functions $f_{c_{1}}=$ $(a+b \sqrt[4]{\beta})$ and $f_{c_{2}}=(c-b \sqrt[4]{\beta})$, where $a, b, c \in \mathbb{F}_{p^{2}}$, the $f_{c_{1}} f_{c_{2}}$ multiplication is unrolled as;

$$
(a+b \sqrt[4]{\beta})(c-b \sqrt[4]{\beta})=a c-b^{2} \sqrt{\beta}+b(c-a) \sqrt[4]{\beta}
$$

Note that here $(c-a) \in \mathbb{F}_{p}$. We can also take advantage of the form of the $a c$ multiplication, where;

$$
a c=(e+f \sqrt{\beta})(g+f \sqrt{\beta})=e g-2 f^{2}+f(e+g) \sqrt{\beta}
$$

So the total cost is $2 M+S$ for the ac multiplication, plus $2 M+2 M$ for the overall multiplication, which results in $6 M+S$ instead of the general $9 M$. When this technique is implemented, we find that although the denominator elimination method is theoretically slightly faster, the performance of denominator elimination and Algorithm 2 is roughly the same, for the genus 2 case under consideration. However, we suggest that Algorithm 2 is a more natural algorithm to use in practice, as it is does not require constructing customised multiplication routines, such as those given in this section.

\subsection{Lucas Exponentiation}

As detailed in algorithm 1, the final exponentiation is split into two parts. The first part can be computed with a conjugation and division, and then Lucas exponentiation, as detailed in the paper by Scott and Barreto 28, is used for the $\left(p^{2}+1\right) / r$ exponentiation. It is also possible to write the $\left(p^{2}+1\right) / r$ exponentiation to the base $p$, and take advantage of the Frobenius endomorphism [16]. However, according to Granger et al. [14, it is faster to use the Lucas sequence approach for curves of low embedding degree.

\subsection{Coding Issues}

We use MIRACL 24] to provide the cryptographic primitives needed. In particular, we make use of special assembly-language routines that MIRACL provides, which can be used when working with moduli with a fixed number of bits. All of the implementation was written in $\mathrm{C} / \mathrm{C}++$ and timed on a Pentium IV, $2.8 \mathrm{Ghz}$.

\subsection{Theoretical Analysis}

Here we analyse the theoretical cost of computing the Tate pairing. Firstly, we reproduce Choie and Lee's analysis, for the sake of comparison. They estimate the cost of computing the Tate pairing (minus the final exponentiation) as

$$
\log _{2}(r)\left(T_{D}+T_{c}+T_{d}+2 T_{s k}+2 T_{m k}\right)+0.5 \log _{2}(r)\left(T_{A}+T_{c}+T_{d}+2 T_{m k}\right)
$$

where $T_{D}$ is the cost of doubling a general divisor, $T_{A}$ is the cost of adding two general divisors, $T_{c}$ and $T_{d}$ the cost of evaluating at the rational functions $c$ and 
$d$, and $T_{s k}$ and $T_{m k}$ the cost of squaring and multiplying respectively in $\mathbb{F}_{p^{k}}$. Note that the authors assume that there will be $r / 2$ additions to be performed, as they employ a random subgroup order with no special conditions.

Let $S$ be a squaring over $\mathbb{F}_{p}$ and let $M$ be a multiplication over $\mathbb{F}_{p}$. Choie and Lee then define $T_{D}=1 I+23 M+5 S$ and $T_{A}=1 I+23 M+2 S$, using their explicit formulae for calculating the group law, and $T_{c}+T_{d}$ as $22 M+5 S$, with an initial precompution of $8 M+3 S$. Finally, they define $T_{s k}=8 M$ and $T_{m k}=9 M$. The total cost then of computing $\langle P, Q\rangle_{r}$ given by Choie and Lee, assuming a subgroup order of size $\log _{2}(r) \approx 160$, is $240 I+17688 M+2163 S$.

We now give the theoretical cost of computing $\langle P, Q\rangle_{r}$ using the optimisations given in this paper. Namely, using a more efficient variant of Miller's algorithm, using a subgroup order of low Hamming weight, using more efficient formulae to calculate the group law, evaluating at a degenerate divisor, and implementing finite field arithmetic more efficiently. The cost of computing the Tate pairing in our case is (again without computing the final exponentiation)

$$
\log _{2}(r)\left(T_{D}+T_{c}+T_{d}+T_{s k}+2 T_{m k}\right)+2\left(T_{A}+T_{c}+T_{d}+2 T_{m k}\right)
$$

where $T_{A}$ and $T_{m k}$ are the same as given by Choie and Lee, $T_{D}=1 I+22 M+$ $4 S, T_{c}+T_{d}=18 M$ with a precomputation of $17 M+1 S$, and $T_{s k}=6 M$. Therefore, the total cost using our optimisations comes to $162 I+10375 M+645 S$, a substantial improvement.

\section{$5 \quad$ Experimental Results}

In this section, we give experimental results for computing the Tate pairing using the techniques detailed in this paper for the supersingular genus 2 curve defined over $\mathbb{F}_{p}$, as defined in section 4.1. We will use the three different levels of security defined earlier for testing, namely (160/1024), (192/2048) and (224/4096).

The only condition on our prime-subgroup order $r$ is that it be congruent to 5 $\bmod 8$. $r$, and not $r^{2}$, must divide the order of the Jacobian, $p^{2}+1$. The prime $p$ must be congruent to $5 \bmod 8$, and also congruent to 2 or $3 \bmod 5$, for reasons stated earlier. The parameters used are detailed in appendix A

Table 3 details the timings for the implementation of the Tate pairing for the (160/1024) security level, table 4 is for the (192/2048) case, and table 5 is for the (224/4096) case. There are four cases in each table. The first is evaluating using a single point. The second case is evaluating at the more general two points, which is the case when the divisor is the sum of two rational points. The third case uses Mumford representation, instead of keeping the points separate. This case has the advantage that it also handles the case when the points on the divisor are defined over a larger field. All these cases use algorithm 2. The fourth cases are timings that are reported in 27] using elliptic curves, with the equivalent level of security.

In the Choie and Lee paper [8], they give implementations of the pairing that range between 500 and $600 \mathrm{~ms}$ on a Pentium IV 2 Ghz for a (160/1024) bit security level. However, as seen in table 3. our timings far outperform this. These timings indicate that genus 2 pairings over prime fields are valid candidates for 
Table 3. Running times - (160/1024) security level

\begin{tabular}{lll}
\hline case description & pairing time $(\mathrm{ms})$ \\
\hline 1 & evaluating at degenerate divisor & 16 \\
2 & evaluating at general divisor & 20.7 \\
3 & evaluating using Mumford rep & 20.45 \\
4 & elliptic curve timing [27] & 8.9 \\
\hline
\end{tabular}

Table 4. Running times - (192/2048) security level

\begin{tabular}{lll}
\hline case description & pairing time $(\mathrm{ms})$ \\
\hline 1 & evaluating at degenerate divisor & 49 \\
2 & evaluating at general divisor & 62 \\
3 & evaluating using Mumford rep & 61 \\
4 & elliptic curve timing [27] & 20.5 \\
\hline
\end{tabular}

Table 5. Running times - (224/4096) security level

\begin{tabular}{lll}
\hline case description & pairing time (ms) \\
\hline 1 & evaluating at degenerate divisor & 183 \\
2 & evaluating at general divisor & 232 \\
3 & evaluating using Mumford rep & 229 \\
4 & evaluating at degenerate divisor (denom elim) & 175 \\
\hline
\end{tabular}

practical implementations. However, as can be seen from the tables, the elliptic cases are roughly twice as fast as the genus 2 timings. This is what we would expect, due to the more complicated group law in the genus 2 case.

\section{Conclusion}

We have introduced a new variant of Miller's algorithm to compute the Tate pairing for curves of even embedding degree, by showing how divisions can always be eliminated. This algorithm is not as fast as using denominator elimination in the general case, but can be faster when working with curves of genus greater than one and distortion maps of a certain form.

We have implemented the Tate pairing on supersingular genus 2 curves over prime fields, detailed various optimisations, and showed how genus 2 curves over prime fields are valid candidates for pairing implementation. In particular, our timings are the fastest reported in the literature to date by a considerable margin.

\section{Acknowledgements}

We would like to thank Steven Galbraith and Caroline Sheedy for comments on this paper, and Noel McCullagh for pointing out an error in a previous draft. We would also like to thank the anonymous referees for their comments. 


\section{References}

1. Barreto, P.S.L.M., Galbraith, S.D., Ó hÉigeartaigh, C., Scott, M.: Pairing computation on supersingular abelian varieties. Cryptology ePrint Archive, Report, 2004/375 (2004), Available from http://eprint.iacr.org/2004/375

2. Barreto, P.S.L.M., Kim, H.Y., Lynn, B., Scott, M.: Efficient algorithms for pairingbased cryptosystems. In: Yung, M. (ed.) CRYPTO 2002. LNCS, vol. 2442, pp. 354-368. Springer, Heidelberg (2002)

3. Barreto, P.S.L.M., Lynn, B., Scott, M.: On the selection of pairing-friendly groups. In: Matsui, M., Zuccherato, R.J. (eds.) SAC 2003. LNCS, vol. 3006, pp. 17-25. Springer, Heidelberg (2004)

4. Blake, I.F., Seroussi, G., Smart, N.P.: Advances in elliptic curve cryptography. Cambridge (2005)

5. Boneh, D., Franklin, M.: Identity-based encryption from the weil pairing. SIAM Journal of Computing 32(3), 586-615 (2003)

6. Cantor, D.G.: Computing in the jacobian of a hyperelliptic curve. Mathematics of Computation 48(177), 95-101 (1987)

7. Choie, Y., Jeong, E., Lee, E.: Supersingular hyperelliptic curves of genus 2 over finite fields. Journal of Applied Mathematics and Computation 163(2), 565-576 (2005)

8. Choie, Y., Lee, E.: Implementation of tate pairing on hyperelliptic curves of genus 2. In: Lim, J.-I., Lee, D.-H. (eds.) ICISC 2003. LNCS, vol. 2971, pp. 97-111. Springer, Heidelberg (2004)

9. Duursma, I., Lee, H.-S.: Tate pairing implementation for hyperelliptic curves $y^{2}=$ $x^{p}-x+d$. In: Laih, C.-S. (ed.) ASIACRYPT 2003. LNCS, vol. 2894, pp. 111-123. Springer, Heidelberg (2003)

10. Frey, G., Lange, T.: Fast bilinear maps from the tate-lichtenbaum pairing on hyperelliptic curves. In: Hess, F., Pauli, S., Pohst, M. (eds.) Algorithmic Number Theory VII. LNCS, vol. 4076, pp. 466-479. Springer, Heidelberg (2006)

11. Frey, G., Rück, H.-G.: A remark concerning $m$-divisibility and the discrete logarithm problem in the divisor class group of curves. Mathematics of Computation 62(206), 865-874 (1994)

12. Galbraith, S., Harrison, K., Soldera, D.: Implementing the tate pairing. In: Fieker, C., Kohel, D.R. (eds.) Algorithmic Number Theory Symposium - ANTS V. LNCS, vol. 2369, pp. 324-337. Springer, Heidelberg (2002)

13. Galbraith, S.D., Paterson, K.G., Smart, N.P.: Pairings for cryptographers. Cryptology ePrint Archive, Report 2006/165 (2006), http://eprint.iacr.org/2006/165

14. Granger, R., Page, D., Smart, N.P.: High security pairing-based cryptography revisited. In: Hess, F., Pauli, S., Pohst, M. (eds.) Algorithmic Number Theory Symposium - ANTS VII. LNCS, vol. 4076, pp. 480-494. Springer, Heidelberg (2006)

15. Ó hÉigeartaigh, C.: Speeding up pairing computation (2005), http://eprint.iacr.org/2005/293

16. Hu, L., Dong, J-W., Pei, D-Y.: Implementation of cryptosystems based on tate pairing. Journal of Computer Science and Technology 20(2), 264-269 (2005)

17. Kobayashi, T., Aoki, K., Imai, H.: Efficient algorithms for tate pairing. IEICE Transactions Fundamentals, E89-A(1) (January 2006)

18. Lange, T.: Formulae for arithmetic on genus 2 hyperelliptic curves. Applicable Algebra in Engineering, Communication and Computing 15(5), 295-328 (2005) 
19. Lenstra, A.K., Verheul, E.R.: Selecting cryptographic key sizes. Journal of Cryptology 14(4), 255-293 (2001)

20. Miller, V.S.: Short programs for functions on curves. Unpublished manuscript (1986), http://crypto.stanford.edu/miller/miller.pdf

21. Miller, V.S.: The weil pairing and its efficient calculation. Journal of Cryptology 17(4), 235-261 (2004)

22. Miyamoto, Y., Doi, H., Matsuo, K., Chao, J., Tsuji, S.: A fast addition algorithm of genus two hyperelliptic curve. In: Symposium on Cryptography and Information Security - SCIS 2002, pp. 497-502.

23. Ribenboim, P.: Classical Theory of Algebraic Numbers. Springer, Heidelberg (2001)

24. Scott, M.: Miracl (multiprecision integer and rational arithmetic $\mathrm{c} / \mathrm{c}++$ library). Available from http://indigo.ie/ mscott/

25. Scott, M.: Faster identity based encryption. Electronics Letters 40(14), 861 (2004)

26. Scott, M.: Computing the tate pairing. In: Menezes, A.J. (ed.) CT-RSA 2005. LNCS, vol. 3376, pp. 293-304. Springer, Heidelberg (2005)

27. Scott, M.: Scaling security in pairing-based protocols. Cryptology ePrint Archive, Report, 2005/139 (2005), http://eprint.iacr.org/2005/139

28. Scott, M., Barreto, P.: Compressed pairings. In: Franklin, M. (ed.) CRYPTO 2004. LNCS, vol. 3152, pp. 140-156. Springer, Heidelberg (2004)

29. Smith, P., Skinner, C.: A public-key cryptosystem and a digital signature system based on the lucas function analogue to discrete logarithms. In: Safavi-Naini, R., Pieprzyk, J.P. (eds.) ASIACRYPT 1994. LNCS, vol. 917, pp. 357-364. Springer, Heidelberg (1995)

30. Solinas, J.: Generalized mersenne numbers. Technical Report CORR 99-39, University of Waterloo (1999), Available from http://www.cacr.math.uwaterloo.ca/ techreports/1999/corr99-39.pdf

\section{A Curve Parameters}

Here we give the subgroup and prime field parameters that were used for the different security levels;

$r=2^{159}+2^{17}+1$

$p=63324531451181148200275171731203125718855624493339065310878459331886717065893$

192/2048 security level:

$r=2^{191}+2^{2}+1$

$p=89284651228083788426899503684145515482879124715345625109737480602016411174689$

53363599067244027908076232256944699958875614648564192943960634648749730387013

224/4096 security level:

$r=2^{223}+2^{13}+1$

$p=15572288413151584018732355885170470078314521100905501866179797721305996406660$

92216915248013505987797528664804210783695074492197917546846433974048512730952

93761493705843127836052457915167872334351960770506641541305942224943595487772

602516676106413200532581353024750990143717859982402535061826066311255496083453 


\section{B Doubling Formulae}

Table 6. Formulae for doubling when $\operatorname{deg} u_{1}=2, \operatorname{gcd}\left(u_{1}, 2 v_{1}\right)=1$ )

\begin{tabular}{|c|c|c|}
\hline \multirow{3}{*}{\begin{tabular}{|c|} 
Input \\
Outpu \\
Step \\
1
\end{tabular}} & $\begin{array}{l}D_{1}=\left[u_{1}, v_{1}\right] \text { where } u_{1}=x^{2}+u_{11} x+u_{10}, v_{1}=v_{11} x+v_{10}, f=x^{5}+a \\
D_{3}=\left[u_{3}, v_{3}\right], l(x) \text { such that } D_{3}+\operatorname{div}\left((y-l) / u_{3}\right)=2 D_{1} .\end{array}$ & \\
\hline & Expression & Cost \\
\hline & $\frac{\text { Compute } \tilde{v_{1}} \equiv\left(2 v_{1}\right)\left(\bmod u_{1}\right)=v_{11} x+v_{10}}{\frac{v_{11}}{\tilde{v_{1}}}=2 v_{11}, \tilde{v_{10}}=2 v_{10}}$ & \\
\hline 2 & $\begin{array}{l}\frac{\text { Compute } r=\operatorname{res}\left(u_{1}, \tilde{v_{1}}\right)}{w_{0}=v_{11}^{2}, w_{1}=u_{11}^{2}, w_{2}}=4 w_{0}, w_{3}=u_{11} v_{11}, \\
r=u_{10} w_{2}+v_{10}\left(\tilde{v_{10}}-w_{3}\right)\end{array}$ & $2 S+3 M$ \\
\hline 3 & $\begin{array}{l}\text { Compute almost inverse of } i n v^{\prime}=r\left(2 v_{1}\right)^{-1}\left(\bmod u_{1}\right) \\
i n v_{1}^{\prime}=-v_{11}, i n v_{0}^{\prime}=v_{10}-w_{3}\end{array}$ & \\
\hline 4 & $\begin{array}{l}\text { Compute } k^{\prime}=\frac{F-v_{1}^{2}}{u_{1}}\left(\bmod u_{1}\right)=k_{1}^{\prime} x+k_{0}^{\prime} \\
w_{3}=w_{1}, w_{4}=2 u_{10}, k_{1}^{\prime}=2 w_{1}+w_{3}-w_{4} \\
k_{0}^{\prime}=u_{11}\left(2 w_{4}-w_{3}\right)-w_{0}\end{array}$ & $1 \mathrm{M}$ \\
\hline 5 & $\begin{array}{l}\text { Compute } s^{\prime}=k^{\prime} i n v^{\prime}\left(\bmod u_{1}\right) \\
w_{0}=k_{0}^{\prime} i n v_{0}^{\prime}, w_{1}=k_{1}^{\prime} i n v_{1}^{\prime} \\
s_{1}^{\prime}=v_{10} k_{1}^{\prime}-v_{11} k_{0}^{\prime}, s_{0}^{\prime}=w_{0}-u_{10} w_{1} \\
\text { If } s_{1}^{\prime}=0 \text { then goto step } 6^{\prime}\end{array}$ & $5 \mathrm{M}$ \\
\hline 6 & $\begin{array}{l}\text { Compute } s=s_{1} x+s_{0} \text { and } s_{1}^{-1} \\
w_{1}=\left(r s_{1}^{\prime}\right)^{-1}, w_{2}=s_{1}^{\prime} w_{1}, w_{3} \\
s_{1}=s_{1}^{\prime} w_{2}, s_{0}=s_{0}^{\prime} w_{2}\end{array}$ & $1 \mathrm{I}, 1 \mathrm{~S}, 5 \mathrm{M}$ \\
\hline 7 & $\begin{array}{l}\text { Compute } l(x)=s u_{1}+v_{1}=s_{1} x^{3}+l_{2} x^{2}+l_{1} x+l_{0} \\
l_{2}=s_{1} u_{11}+s_{0}, l_{0}=s_{0} u_{10}+v_{10} \\
l_{1}=\left(s_{1}+s_{0}\right)\left(u_{11}+u_{10}\right)-s_{1} u_{11}-s_{0} u_{10}+v_{11}\end{array}$ & $3 \mathrm{M}$ \\
\hline 8 & $\begin{array}{l}\text { Compute } u^{\prime}=\operatorname{monic}\left(\frac{F-l^{2}}{u_{1}^{2}}\right)=x^{2}+u_{31} x+u_{30} \\
u_{30}=w_{3}\left(2 v_{11}+w_{3}\left(2 u_{11}+s_{0}^{2}\right)\right. \\
u_{31}=2 s_{0}-w_{3}\end{array}$ & $1 \mathrm{~S}+2 \mathrm{M}$ \\
\hline 9 & $\begin{array}{l}\text { Compute } v_{3}=-l\left(\bmod u_{3}\right)=v_{31} x+v_{30} \\
w_{1}=u_{31}, u_{31}=w_{3} u_{31}, w_{3}=l_{2}-w_{1}, w_{3}=u_{30} w_{2} \\
v_{31}=\left(u_{31}+u_{30}\right)\left(w_{2}+s_{1}\right)-w_{3}-w_{1}-l_{1}, v_{30}=w_{3}-l_{0}\end{array}$ & $3 \mathrm{M}$ \\
\hline & & $1 \mathrm{I}, 4 \mathrm{~S}, 22 \mathrm{M}$ \\
\hline $6^{\prime}$ & 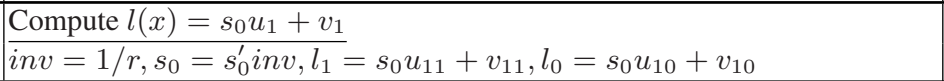 & $1 \mathrm{I}+3 \mathrm{M}$ \\
\hline $7^{\prime}$ & $\begin{array}{l}\text { Compute } u_{3}=\operatorname{monic}\left(\frac{F-l^{2}}{u_{1}^{2}}\right)=x+u_{30} \\
u_{30}=-2 u_{11}-s_{0}^{2}\end{array}$ & $1 \mathrm{~S}$ \\
\hline 8 ' & $\frac{\text { Compute } v_{3}=-l\left(\bmod u_{3}\right)=v_{30}}{v_{30}=u_{30}\left(l_{1}-u_{30} s_{0}\right)-l_{0}}$ & $2 \mathrm{M}$ \\
\hline & & $1 \mathrm{I}, 3 \mathrm{~S}, 14 \mathrm{M}$ \\
\hline
\end{tabular}

